\title{
A factorized Regge-pole model for deeply virtual Compton scattering
}

M. Capua, S. Fazio, R. Fiore

Dipartimento di Fisica, Università della Calabria

Istituto Nazionale di Fisica Nucleare, Gruppo collegato di Cosenza

I-87036 Arcavacata di Rende, Cosenza, Italy

\section{Jenkovszky*}

Bogolyubov Institute for Theoretial Physics, Kiev-143, Ukraine

E-mail: jenk@bitp.kiev.ua

\section{F. Paccanoni}

Dipartimento di Fisica, Università di Padova,

Istituto Nazionale di Fisica Nucleare, Sezione di Padova

via Marzolo 8, I-35131, Italy

\begin{abstract}
A Regge-factorized model for deeply virtual Compton scattering (DVCS), involving a new variable $z$ connecting $t$ (squared transferred momentum) and $Q^{2}$ (photon virtuality), is suggested. The transition from the exclusive DVCS to the inclusive deep inelastic scattering (DIS) is realized explicitly. Physical meaning is given to the adjustable parameters. From the imaginary part of the scattering amplitude generalized parton distributions (GPD) can be calculated.
\end{abstract}

DIFFRACTION 2006 - International Workshop on Diffraction in High-Energy Physics September 5-10 2006

Adamantas, Milos island, Greece

*Speaker. 


\section{Introduction}

Generalized parton distributions (GPDs) combine our knowledge about the one-dimensional parton distribution in the longitudinal momentum with the impact-parameter, or transverse distribution of matter in a hadron or nucleus [1,2]. It is an ambitious program to access the spatial distribution of partons in the transverse plane and thus to provide a 3-dimensional picture of the nucleon (nucleus) (see Ref. [3] and earlier references therein). This program involves various approaches, including perturbative QCD, Regge poles, lattice calculations, etc. The main problem is that, while the partonic subprocess can be calculated perturbatively, the calculation of GPDs require non-perturbative methods. GPDs enter hard exclusive processes, such as deeply virtual Compton scattering (DVCS); however, they cannot be measured directly but instead appear in convolution integrals, that cannot be easily converted. Hence the strategy is to guess the GPD, based on various theoretical constraints, and then compare it with the data. In the first approximation, the GPD is proportional to the imaginary part of a DVCS amplitude, therefore, the knowledge (or experimental reconstruction) of the DVCS amplitude may partly resolve the problem, provided the phase of the DVCS amplitude is also known. In other words, a GPD can be viewed as the imaginary part of an antiquark-nucleon scattering amplitude, or a quark-nucleon amplitude in the $u$ channel. Interest in deeply virtual Compton scattering (DVCS) $e p \rightarrow e \gamma p$ is triggered by the prospects to use it as a tool in studies of GPDs.

DVCS is being measured by different experimental groups in various kinematical regions: at JLab, at relatively low energies, close to the resonance region, but with high intensity beams (statistics) and for various spin configurations, at CERN, by the COMPASS Collaboration, with a fixed muon target, and at HERA - both with a fixed target (HERMES) and in ep collider mode (H1 and ZEUS).

At HERA the DVCS cross-section has been measured $[4,5]$, in diffractive $e p$ interactions, as a function of $Q^{2}, W$ and $t$ that are respectively the photon virtuality, the invariant mass of the $\gamma^{*} p$ system and the squared 4-momentum transferred at the proton vertex; the diagram in Fig. ??a shows the production of a real photon at HERA.

The $Q^{2}$ evolution of the DVCS amplitude has been studied in several papers, mainly in the context of perturbative quantum chromodynamics (QCD) $[6,7]$ and recently in [8]. The $t$ dependence in many papers was introduced by a simple factorized exponential in $t$, which however contradicts the Regge pole theory. Since the electron-proton scattering at HERA is dominated by a single photon exchange, the calculation of the DVCS scattering amplitude reduces to that of the $\gamma^{*} p \rightarrow \gamma p$ amplitude, which at high energies, in the Regge pole approach, is dominated by the exchange of positive-signature Reggeons, associated with the Pomeron and the $f$-trajectories [9]. Fig. ??b shows this DVCS in a Regge-factorized form. In this figure $q_{1,2}$ are the four-momenta of the incoming and outgoing photons, $p_{1,2}$ are the four-momenta of the incoming and outgoing protons, $r$ is the four-momentum of the Reggeon exchanged in the $t$ channel, $r^{2}=t=\left(q_{1}-q_{2}\right)^{2}$ and $s=W^{2}=\left(q_{1}+p_{1}\right)^{2}$ is the squared centre-of-mass energy of the incoming system.

Unless specified (as in the deep inelastic scattering (DIS) limit, discussed in Sec. 3 and for vector meson production), $q_{2}^{2}=0$, and hence, for brevity, $q_{1}^{2}=-Q^{2}$. In the upper vertex $V_{1}$, Fig. 1b, a virtual photon with 4-momentum $q_{1}$, and a Reggeon (e.g. Pomeron) with 4-momentum $r$, enter and a real photon, with 4-momentum $q_{2}=q_{1}+r$ (or a vector meson) appears in the final 

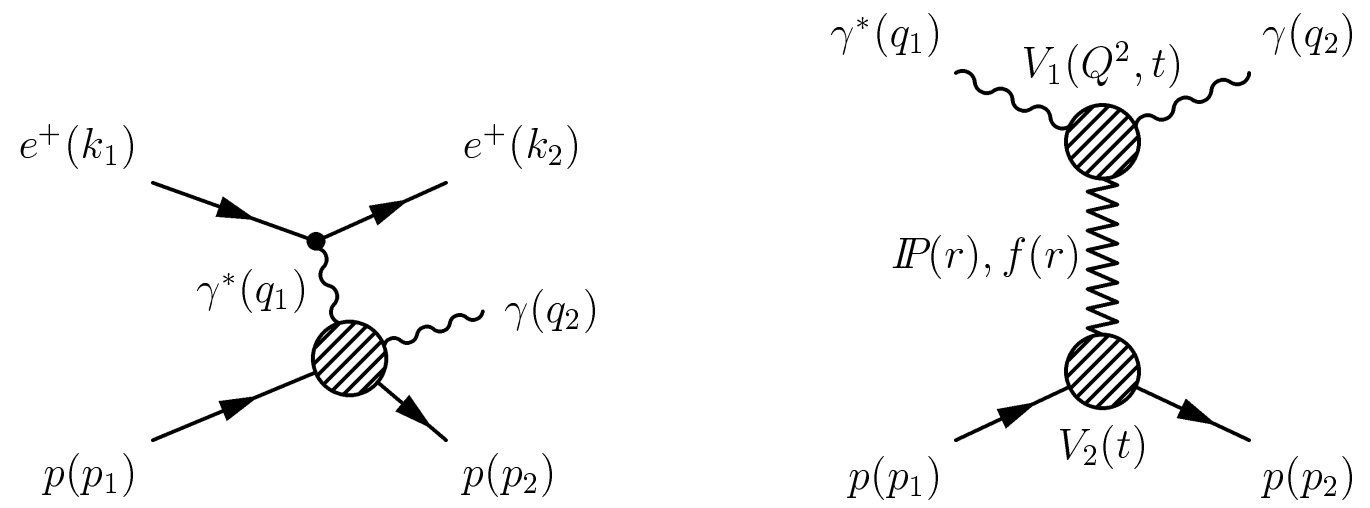

Figure 1: Reaction ep $\rightarrow$ ep $\gamma$ resulting in deeply virtual Compton scattering (DVCS) (left panel) and a DVCS amplitude in a Regge-factorized form (right panel).

state as an outgoing particle. The vertex $V_{1}$ depends on all the possible invariants constructed with the above 4-momenta, $V_{1}\left[q_{1}^{2}, r^{2}, q_{1} \cdot r\right]$, where $r^{2}=t \leq 0, q_{1}^{2}=-Q^{2} \leq 0$. The three invariants are not independent since the mass-shell condition for the outgoing photon, $q_{2}^{2}=\left(q_{1}+r\right)^{2}=0$, provides the relation

$$
q_{1} \cdot r=\frac{-q_{1}^{2}-r^{2}}{2}=\frac{Q^{2}-t}{2}
$$

Hence, the vertex can be considered as a function of the invariants $\left[Q^{2}, q_{1} \cdot r\right]$ or $\left[t, q_{1} \cdot r\right]$. This does not mean that the variables cannot appear separately but it could also happen that $q_{1} \cdot r$ becomes a scaling variable, and consequently the vertex will finally depend on $q_{1} \cdot r$ only. It depends on the dynamics of the process and, for the moment, we prefer to keep $t$, apart from $Q^{2}$, as the second independent variable.

Electroproduction of a vector meson gives another example since in this case $\left(q_{1}+r\right)^{2}=M_{V}^{2}$, and the variable $q_{1} \cdot r$ becomes

$$
q_{1} \cdot r=\frac{M_{V}^{2}-q_{1}^{2}-r^{2}}{2}=\frac{M_{V}^{2}+Q^{2}-t}{2} .
$$

The interplay of the $Q^{2}$ - and $t$-dependence in the DVCS amplitude was recently discussed in Ref. [10], where the existence of a new, universal variable $z$ was suggested. The basic idea is that $Q^{2}$ and $t$, both having the meaning of the squared mass of a virtual particle (photon or Reggeon), should be treated on the same footing, by means a new variable, defined as

$$
z=q_{1}^{2}+t=-Q^{2}+t
$$

in the same way as the vector meson mass squared is added to the squared photon virtuality, giving $\tilde{Q}^{2}=Q^{2}+M_{V}^{2}$ in the case of vector meson electroproduction [11].

In this contribution we present an explicit model for DVCS with $Q^{2}$ - and $t$-dependences determined by the $\gamma^{*} P \gamma$ vertex. We suggest the use of the new variable defined in Eq. (1.3) with its possible generalization to vector meson electroproduction,

$$
z=t-\left(Q^{2}+M_{V}^{2}\right)=t-\tilde{Q}^{2}
$$


or virtual photon (lepton pair) electroproduction,

$$
z=t-\left(Q_{1}^{2}+Q_{2}^{2}\right),
$$

where $Q_{2}^{2}=-q_{2}^{2}$. Differently from Ref. [10], here we introduce the new variable only in the upper, $\gamma^{*} P \gamma$ vertex, to which the photons couple.

\section{A DVCS amplitude}

Similar to [8], we consider only the helicity conserving amplitude.

According to the ideas of Regge-factorization, Fig. ??b, this DVCS amplitude can be written as

$$
A\left(s, t, Q^{2}\right)_{\gamma^{*} p \rightarrow \gamma p}=-A_{0} V_{1}\left(t, Q^{2}\right) V_{2}(t)\left(-i s / s_{0}\right)^{\alpha(t)},
$$

where $A_{0}$ is a normalization factor, $V_{1}\left(t, Q^{2}\right)$ is the $\gamma^{*} P \gamma$ vertex, $V_{2}(t)$ is the $p P p$ vertex and $\alpha(t)$ is the exchanged Regge trajectory. The scattering amplitude is a sum of all possible Regge exchanges, with positive signature in reactions under consideration. In the HERA collider region, the main contribution comes from the Pomeron (P) and $f$-meson trajectories, the Pomeron being dominant. In a future detailed analyses with more data points available, these (and other sub-leading) Regge pole exchanges can be appended by their interference (possible cuts), making however the situation much more complicated. For this reason, we collect all these Regge exchanges in a single one, called and effective trajectory, which we assume to be of a logarithmic form:

$$
\alpha(t)=\alpha(0)-\alpha_{1} \ln \left(1-\alpha_{2} t\right)
$$

Such a trajectory is nearly linear for small $|t|$, reproducing the forward cone of the differential cross section, while its logarithmic asymptotics provides for the large-angle scaling behavior $[13,16]$, typical of hard collisions at small distances, with power-law fall-off in $|t|$, and obeying quark counting rules $[14,15]$. Let us remind that we are referring to the dominant Pomeron contribution plus a secondary trajectory, e.g. the $f$-Reggeon. Being aware of the importance of this sub-leading contribution at HERA energies (even more so in interpreting fixed-target data of HERMES), nevertheless we cannot afford the duplication of the number of free parameters, therefore we include it effectively by rescaling the parameters. Ultimately, the Pomeron and the $f$-Reggeon have the same functional form, differing only in the values of their parameters. Furthermore, the Pomeron [18] itself is unlikely to be a single term, so instead of including several Regge terms with many free parameters, it may be reasonable to comprise them in a single term, called effective Reggeon or effective Pomeron, depending on the kinematical region of interest. Although the parameters of this effective Reggeon (Pomeron) (e.g. its intercept and slope) can be close to the true one (whose form is at best a convention), for the above reason they never should be taken for granted.

For convenience, and following the arguments based on duality (see Ref. [17] and references therein), the $t$ dependence of the $p P p$ vertex is introduced via the $\alpha(t)$ trajectory: $V_{2}(t)=e^{b \alpha(t)}$ where $b$ is a parameter. A generalization of this concept is applied also to the upper, $\gamma^{*} P \gamma$ vertex by introducing the trajectory

$$
\beta(z)=\alpha(0)-\alpha_{1} \ln \left(1-\alpha_{2} z\right)
$$


where the value of the parameter $\alpha_{2}$ may be different in $\alpha(t)$ and $\beta(z)$ (a relevant check will be possible when more data will be available). Hence the scattering amplitude (6), with the correct signature, becomes

$$
A\left(s, t, Q^{2}\right)_{\gamma^{*} p \rightarrow \gamma p}=-A_{0} e^{b \alpha(t)} e^{b \beta(z)}\left(-i s / s_{0}\right)^{\alpha(t)}=-A_{0} e^{(b+L) \alpha(t)+b \beta(z)},
$$

where $L \equiv \ln \left(-i s / s_{0}\right)$.

The model contains a limited number of free parameters. Moreover, most of them can be estimated a priori. The product $\alpha_{1} \alpha_{2}$ is just the forward slope $\alpha^{\prime}$ of the Reggeon $\left(\approx 0.2 \mathrm{GeV}^{-2}\right.$ for the Pomeron, but much higher for $f$ and/or for an effective Reggeon) ${ }^{1}$. The value of $\alpha_{1}$ can be estimated from the large-angle quark counting rules $[13,14,15]$. In a dual model, the value of $\alpha_{1}$ can be estimated from the large-angle quark counting rules since, for large $t\left(|t|>>1 \mathrm{GeV}^{2}\right)$, the amplitude goes roughly (for more details see Refs. [16]) as $\sim e^{-\alpha_{1} \ln (-t)}=(-t)^{-\alpha_{1}}$. In this case the power $\alpha_{1}$ is related to the number of quarks in a collision [13,14, 15].The amplitude (2.4), however, does not scale exactly since it contains an extra $s$-dependent factor, for which reason the above arguments are only approximate. We made trials with $\alpha_{1}$ ranging from 1 to 4 and found little variation of the resulting fits, moreover the best $\chi^{2}$ was achieved with $\alpha_{1}$ close 1 . Following this indication, we fixed $\alpha_{1}$ at 1 , thus reducing the number of the free parameters by one.

We fix the intercept of our effective Reggeon, dominated by the Pomeron, at $\alpha(0)=1.25$, as an average over the soft+hard Pomerons ${ }^{2}$. Finally, there are good reasons to set the value of the scaling parameter equal to the inverse slope, $s_{0}=1 / \alpha^{\prime}$.

The above values of the parameters should not be taken for granted, rather they should be considered as starting points (inputs) in fitting the model to the data (see [19]).

From Eq. (2.4) the slope of the forward cone is

$$
B\left(s, Q^{2}, t\right)=\frac{d}{d t} \ln |A|^{2}=2\left[b+\ln \left(\frac{s}{s_{0}}\right)\right] \frac{\alpha^{\prime}}{1-\alpha_{2} t}+2 b \frac{\alpha^{\prime}}{1-\alpha_{2} z},
$$

which, in the forward limit, $t=0$ reduces to

$$
B\left(s, Q^{2}\right)=2\left[b+\ln \left(\frac{s}{s_{0}}\right)\right] \alpha^{\prime}+2 b \frac{\alpha^{\prime}}{1+\alpha_{2} Q^{2}} .
$$

Thus, the slope shows shrinkage in $s$, as expected, and observed in all diffractive processes, and antishrinkage in $Q^{2}$ - a novel feature in Regge-type models. Shrinkage in $Q^{2}$, and the $Q^{2}$-evolution on the whole, is a basic feature of perturbative QCD. Here it is correlated by the form of the Regge trajectory and the $t-$ dependence of the amplitude. This correlation strongly constrains the model.

Detailed fits of this model to the DVCS data from HERA can be found in [19].

\footnotetext{
${ }^{1}$ As emphasized in a number of papers, e.g. in Ref. [12], the wide-spread prejudice of the flatness of the Pomeron in electroproduction is wrong for at least two reasons: one is that it was deduced by fitting data to a particular effective Reggeon (see the relevant discussion above) and the second is that the Pomeron is universal, and its nonzero slope is well known from hadronic reactions.

${ }^{2}$ This is an obvious simplification and we are fully aware of the variety of alternatives for the energy dependences, e.g. that of a dipole Pomeron, as in Ref. [17], a soft plus a hard one, as e.g. in Ref. [6]. Ultimately, from QCD's BFKL equation [18] an infinite number of Pomeron singularities follows unless simplifications are used. For the present study in term of the new, $z$, variable the simplest supercritical Pomeron [6] with an effective intercept is suitable.
} 
As already mentioned, with a replacement $\tilde{Q}^{2}=Q^{2}+M_{V}^{2}$, the model can be applied also to vector meson elctroproduction.

\section{Photoproduction- and DIS limits}

In the $Q^{2} \rightarrow 0$ limit the Eq. (2.4) becomes

$$
A(s, t)=-A_{0} e^{2 b \alpha(t)}\left(-i s / s_{0}\right)^{\alpha(t)},
$$

where we recognize a typical Regge-behaved photoproduction (or, for $Q^{2} \rightarrow m_{H}^{2}$, on-shell hadronic $(H)$ ) amplitude. The related deep inelastic scattering structure function is recovered by setting $Q_{2}^{2}=Q_{1}^{2}=Q^{2}$ and $t=0$, to get a typical elastic virtual forward Compton scattering amplitude:

$$
A\left(s, Q^{2}\right)=-A_{0} e^{b\left(\alpha(0)-\alpha_{1} \ln \left(1+\alpha_{2} Q^{2}\right)\right)} e^{\left(b+\ln \left(-i s / s_{0}\right)\right) \alpha(0)} \propto-\left(1+\alpha_{2} Q^{2}\right)^{-\alpha_{1}}\left(-i s / s_{0}\right)^{\alpha(0)} .
$$

For not too large $Q^{2}$ the contribution from longitudinal photons is small (it vanishes for $Q^{2}=0$ ). Moreover, at high energies, typical of the HERA collider, the amplitude is dominated by the helicity conserving Pomeron exchange and, since the final photon is real and transverse, the initial one is also transverse - to the extent that helicity is conserved. Hence the relevant structure function is $F_{1}$ that, at leading order, is related to $F_{2}$ by the Callan-Gross relation, to be used in obtaining eq. (3.3).

For $t=0$ (with $x \approx Q^{2} / s$ valid for large $s$ ), the structure function assumes the form:

$$
F_{2}\left(s, Q^{2}\right) \approx \frac{(1-x) Q^{2}}{\pi \alpha_{e}} \mathfrak{I} A\left(s, Q^{2}\right) / s,
$$

where $\alpha_{e}$ is the electromagnetic coupling constant and the normalization is $\sigma_{t}(s)=\frac{4 \pi}{s} \mathfrak{I} A\left(s, Q^{2}\right)$. The resulting structure function has the correct (required by gauge invariance) $Q^{2} \rightarrow 0$ limit and approximate scaling behavior in $x$ for large enough $s$ and $Q^{2}$.

The model fails at large $Q^{2}$, where Bjorken scaling is known to be badly violated. In this domain Regge behavior should be replaced by (or appended with) the DGLAP evolution, as shown, for example, in Ref. [20]. An explicit model interpolating between Regge behavior at small- and intermediate $Q^{2}$ and the approximate solution of the DGLAP equation at large $Q^{2}$ was developed in Ref. [21]. In any case, a "global fit" to DVCS and DIS data would require also the inclusion of both the longitudinal and transverse photons.

\section{From DVCS to GPD}

As shown in $[22,23]$, the imaginary part of the DVCS amplitude, in the first approximation, is proportional to a general parton distribution (GPD). By introducing the skewness variable $\xi=$ $x\left(1+t /\left(2 Q^{2}\right)\right) /\left(2-x+x t / Q^{2}\right)$, the DVCS amplitude (or a GPD) can be written in terms of $s, t, Q^{2}$ or $x, \xi, t$.

The Fourier-Bessel integral

$$
q(x, b) \sim \frac{1}{2 \pi} \int_{0}^{\infty} d \sqrt{-t} J_{0}(b \sqrt{-t}) \mathfrak{I} A(x, t)
$$


provides a mixed representation of longitudinal momentum and transverse position in the infinitemomentum frame.

Numerical calculations of the $x$ (partons' momenta in the longitudinal direction) and $b$ (impact parameter) of the quark distributions with nonlinear Regge trajectories in a related model were made in [3].

\section{Conclusions and discussion}

The model presented in this paper may have two-fold applications. On one hand, it can be used by experimentalists as a guide. The fits to the data could be improved, when more data are available, by accounting for the Pomeron and $f$-Reggeon contributions separately as well as by using expressions for Regge trajectories which take exactly into account analyticity and unitarity. On the other hand, the model can be used to study various extreme regimes of the scattering amplitude in all the three variables it depends on. For that purpose, however, the transition from Regge behavior to QCD evolution at large $Q^{2}$ should be accounted for. A formula interpolating between the two regimes (Regge pole and asymptotic QCD evolution) was proposed in Ref. [21] for $t=0$ only. Its generalization to non zero $t$ values [24] is possible also by applying the ideas and the model presented in this paper. Its applicability in both soft and hard domains can be used to learn about the transition between perturbative (QCD) and non-perturbative (Regge poles) dynamics.

Our approach combines elements of the analytic $S$ - matrix theory, namely Regge poles and duality, known to be efficient for "soft" collisions at large distances, with the small-distance partonic picture. The interface and merge of these seemingly orthogonal approaches may bring new ideas about the transition form perturbative to non-perturbative physics. Much of this information is encoded in the form of the complex Regge trajectories. Recently, explicit models for deeply virtual Compton scattering amplitudes (DVCS) appeared in the literature [25].

In a perspective, one may think of extending the asymptotic Regge-pole model to the lowenergy resonance region by incorporating a dual amplitude, e.g., DAMA. The crossing-symmetric properties of dual amplitudes will make possible the inclusion of the $t>0$ region in DVCS and resulting GPD. It may also help to connect the high-energy (Regge) region with the low-energy (resonance) domain, where new data from JLab is expected.

Independently of the pragmatic use of this model as a instrument to guide experimentalists, given its explicit form, it can be regarded also as an explicit realization of the corresponding principle [?] of exclusive-inclusive connection in various kinematical limits.

Acknowledgments We thank the Organizers of this wonderful meeting for their excellent performance and kind hospitality. The work of L.J. was partly supported by the Ukrainian-Hungarian collaborative Grant 101/2005 of the Ukrainian Ministry of Education and Sciences. 


\section{References}

[1] F. M. Dittes, D. Mueller, D. Robaschik, B. Geyer and J. Horejsi, Phys. Lett. B 209 (1988) 325; D. Mueller, D. Robaschik, B. Geyer, F. M. Dittes and J. Horejsi, Fortsch. Phys. 42 (1994) 101.

[2] X. Ji, Phys. Rev. Lett. 78 (1997) 610; A.V. Radyushkin, Phys. Lett. B 380 (1996) 417; M. Diehl, Phys. Rept. 388 (2003) 41.

[3] L.L. Jenkovszky, arXiv:hep-ph/0607340, submitted to Phys. Rev. D.

[4] H1 Coll.: A. Aktas et al., Eur. Phys. J. C 44 (2005) 1.

[5] ZEUS Coll.: S. Chekanov et al., Phys. Lett. B 573 (2003) 46.

[6] A. Donnachie and H.G. Dosch, Phys. Lett. B 502 (2001) 118.

[7] A. Freund, M. McDermott, M. Strikman, Phys. Rev. D 67 (2003) 036001; L. Favart, M.V.T. Machado, Eur. Phys. J. C19 (2003) 365; V. Guzey, M.V. Polyakov, Eur. Phys. J. C 46 (2006) 151.

[8] D. Muller, arXiv:hep-ph/0605013.

[9] P.D.B. Collins, An Introduction to Regge Theory and High Energy Physics, Cambridge University Press, 1977.

[10] R. Fiore, L. Jenkovszky, V. Magas, and A. Prokudin, Interplay between $Q^{2}-$ and $t-$ dependences in deeply virtual Compton scattering, In the Proceedings of the Crimean conference New Trends in High-Energy Physics, Yalta, 10-17 September 2005.

[11] E.S. Martynov, E. Predazzi, A. Prokudin, Phys. Rev. D 67 (2003) 074023.

[12] R. Fiore, L.L. Jenkovszky, F. Paccanoni and A. Papa, Phys. Rev. D 65 (2002); R. Fiore, L.L. Jenkovszky, F. Paccanoni and A. Prokudin, Phys. Rev. D 68 (2003) 014005.

[13] D.D. Coon, J.F. Gunion, Tran Thanh Van, R. Blankenbecler, Phys. Rev. D 18 (1978) 1451.

[14] V.A. Matveev, R.M. Muradyan, A.N. Tavkhelidze, Lett. Nuovo Cim. 7 (1973) 719.

[15] S. Brodsky and G. Farrar, Phys. Rev. Lett. 31 (1973) 1153.

[16] L.L. Jenkovszky, Z.E. Chikovani, Yad. Fizika 30 (1979) 531 (English translation: Sov. J. Nucl. Phys.); A.I. Bugrij, Z.E. Chikovani, L.L. Jenkovszky, Z. Phys. C, Particles and Fields, 4 (1980) 45; L.L. Jenkovszky, Nucl. Phys. B (Proc. Suppl.) 12 (1990) 317; R. Fiore, L.L. Jenkovszky, V.K. Magas, and F. Paccanoni, Phys. Rev. D 60 (1999) 116003.

[17] L.L. Jenkovszky, Rivista Nuovo Cim. 10 (1987) 1; M. Bertini et al. ibid. 19 (1996) 1.

[18] V.S. Fadin, E.A. Kuraev, L.N. Lipatov, Phys. Lett. B 60 (1975) 50; I.I. Balitsky, L.N. Lipatov, Yad. Fizika 28 (1978) 822.

[19] M. Capua, In these Proceedings; see also: M. Capua, S. Fazio, R. Fiore, L.L. Jenkovszky, and F. Paccanoni, arXiv:hep-ph/0605319, submitted to Phys. Letters B.

[20] L. Csernai et al. Eur. Phys. J. C 24 (2002) 205.

[21] P. Desgrolard, L.L. Jenkovszky, F. Paccanoni, Eur. Phys. J. C 7 (1999) 263.

[22] L.L. Jenkovszky, V.K. Magas, and A. Vall, Physics of Particles and Nuclei, Vol. 36, Suppl.2, 2005, pp. S20-S23.

[23] R. Fiore, L.L. Jenkovszky and V.K. Magas, Nuclear Phys. B (Proc. Suppl.) 146 (2005) 146.

[24] P. Freund and V. Guzey, arXiv:hep-ph/9801388.

[25] A.P. Szczepaniak and J.T. Longedran, Exclusive Eectroproduction and Quark Structure of the Nucleon, arXiv:hep-ph/0604266. 